\title{
Genetic profiles distinguish different types of hereditary ovarian cancer
}

\author{
KATARINA DOMANSKA ${ }^{1}$, SUSANNE MALANDER ${ }^{1}$, JOHAN STAAF $^{1}$, ANNA KARLSSON ${ }^{1}$, \\ $\AA^{\text {AKE BORG }}{ }^{1}$, GÖRAN JÖNSSON ${ }^{1}$ and MEF NILBERT ${ }^{1,2}$ \\ ${ }^{1}$ Institute of Clinical Sciences, Department of Oncology, Lund University, Lund, Sweden \\ ${ }^{2}$ Clinical Research Centre, Hvidovre Hospital, Copenhagen University, Hvidovre, Denmark
}

Received February 5, 2010; Accepted March 29, 2010

DOI: $10.3892 /$ or_00000934

\begin{abstract}
Heredity represents the strongest risk factor for ovarian cancer with disease predisposing mutations identified in $15 \%$ of the tumors. With the aim to identify genetic classifiers for hereditary ovarian cancer, we profiled hereditary ovarian cancers linked to the hereditary breast and ovarian cancer (HBOC) syndrome and the hereditary non-polyposis colorectal cancer (HNPCC) syndrome. Genome-wide array comparative genomic hybridization was applied to $12 \mathrm{HBOC}$ associated tumors with BRCAl mutations and 8 HNPCC associated tumors with mismatch repair gene mutations with 24 sporadic ovarian cancers as a control group. Unsupervised cluster analysis identified two distinct subgroups related to genetic complexity. Sporadic and HBOC associated tumors had complex genetic profiles with an average $41 \%$ of the genome altered, whereas the mismatch repair defective tumors had stable genetic profiles, with an average $18 \%$ of the genome altered. Losses of 4q34, 13q12-q32 and 19p13 were overrepresented in the HBOC subset. Discriminating genes within these regions include BRCA2, FOXO1A and $R B 1$. Gains on chromosomes 17 and 19 characterized the HNPCC tumors, but target genes herein are unknown. The results indicate that HBOC and HNPCC associated ovarian cancer develop along distinct genetic pathways and genetic profiles can thus be applied to distinguish between different types of hereditary ovarian cancer.
\end{abstract}

\section{Introduction}

Heredity is one of the strongest risk factors for ovarian cancer with $10-15 \%$ of the tumors linked to the hereditary breast and ovarian cancer (HBOC) syndrome and 2-4\% to the hereditary non-polyposis colorectal cancer (HNPCC) syndrome (1-3). HBOC is caused by mutations in the BRCAl

Correspondence to: Dr Katarina Domanska, Institute of Clinical Sciences, Department of Oncology, Lund University, 22185 Lund, Sweden

E-mail: katarina.domanska@med.lu.se

Key words: ovarian cancer, heredity, arrayCGH, BRCA1, hereditary non-polyposis colorectal cancer and $B R C A 2$ genes, with the highest (20-40\%) life time risk of ovarian cancer in individuals with $B R C A l$ mutations (4). HBOC associated ovarian cancer typically develops at 50-55 years of age with a predominance of serous adenocarcinomas (5). HNPCC is caused by defective mismatch repair (MMR), primarily due to mutations in the genes $M L H 1, M S H 2$ and MSH6. Female HNPCC family members are at $10-15 \%$ risk of ovarian cancer. These tumors typically develop at an early age; mean 40-45 years, with a predilection for endometrioid, clear cell and mucinous subtypes $(6,7)$.

Refined methods for risk prediction and early tumor detection are needed since late stage diagnosis constitutes a major reason for the poor prognosis of ovarian cancer.

The BRCA1 and BRCA2 genes, as well as the MMR genes, play critical roles in maintenance of genomic stability and repair. BRCA 1 and BRCA 2 are involved in double strand break repair, mainly through interaction with RAD51. BRCA1 inactivation evokes pleiotrophic effects on cell cycle control, transcriptional activation and consequently cells defective for BRCA1 exhibit extensive chromosomal instability (8). In ovarian cancer, the BRCAl genotype is linked to highly malignant tumors, in which no precancerous state has been identified (9). MMR defects interfere with repair of single mismatched base pairs and small nucleotide insertions/deletions. MMR defective tumors generally demonstrate few genetic changes and near diploid karyotypes, also in advanced tumor stages, though data on the genomic profiles of HNPCC associated ovarian cancer are scarce.

Identification of women with hereditary ovarian cancer allows for preventive measures for the more commonly occurring breast cancers in HBOC and colorectal and endometrial cancers in HNPCC $(10,11)$. However, identification of these cases is challenging and a substantial number of individuals with hereditary ovarian cancer escape detection $(12,13)$. We applied high resolution array based comparative genomic hybridization (aCGH) to HBOC and HNPCC associated ovarian cancer with sporadic ovarian cancers as a control group, and demonstrated distinct genetic profiles linked to the underlying type of heredity.

\section{Materials and methods}

Patients and tumors. Epithelial ovarian cancers (borderline tumors excluded) from 24 sporadic cases, 12 BRCA1 mutated 
Table I. Summary of clinicopathological factors.

\begin{tabular}{|c|c|c|c|}
\hline & $\begin{array}{c}\text { HNPCC } \\
\text { tumors } \\
n=8\end{array}$ & $\begin{array}{c}\text { HBOC } \\
\text { tumors } \\
n=12\end{array}$ & $\begin{array}{c}\text { Sporadic } \\
\text { tumors } \\
n=24\end{array}$ \\
\hline $\begin{array}{l}\text { Age } \\
\text { median (range) }\end{array}$ & $47(42-61)$ & $57(42-75)$ & $60(27-78)$ \\
\hline \multicolumn{4}{|l|}{ FIGO stage } \\
\hline I & 3 & 1 & 3 \\
\hline II & 3 & 1 & 1 \\
\hline III & 1 & 8 & 15 \\
\hline IV & 1 & 2 & 5 \\
\hline \multicolumn{4}{|l|}{ Histology } \\
\hline Serous & 1 & 8 & 17 \\
\hline Mucinous & 1 & 0 & 3 \\
\hline Endometroid & 3 & 3 & 4 \\
\hline Clear cell & 3 & 1 & 0 \\
\hline \multicolumn{4}{|l|}{ Differentiation } \\
\hline Well & 1 & 1 & 5 \\
\hline Moderate & 4 & 0 & 5 \\
\hline Poor & 2 & 10 & 13 \\
\hline Unknown & 1 & 1 & 1 \\
\hline
\end{tabular}

cases and 8 cases with mutations in $M L H 1, M S H 2$ and MSH6 were available for the study. Clinical and histopathological data are summarized in Table I. A gynecological pathologist reviewed all diagnoses and four histotypes (serous, mucinous, endometrioid and clear cell adenocarcinomas) were represented (Table I). None of the patients had received preoperative radiotherapy or chemotherapy. The median age at diagnosis was 60 years in the sporadic subset, 57 years in the BRCA1 subset and 47 years in the HNPCC subset. The 24 sporadic tumors were obtained from a population based consecutive series of ovarian cancers, in which mutations in BRCA1 and BRCA2 had been excluded through mutation analysis and retained MMR function had been demonstrated using immunostaining against the MLH1, PMS2, MSH2 and MSH6 proteins $(13,14)$. All BRCAl mutations were classified as disease predisposing and were available from a population based consecutive series of ovarian cancers. The MMR gene mutations were verified using immunohistochemistry and microsatellite analysis and disease predisposing mutations affected $M L H 1$ in one case, $M S H 2$ in three cases and MSH6 in four cases. All ovarian cancers that had developed in Swedish females carrying HNPCC predisposing MMR gene mutations were eligible for the study. Further requirements for all tumors were availability of histopathological material and that DNA of sufficient amount and quality could be obtained. Ethical approval for the study was obtained from the Lund University ethics committee.

DNA extraction, labeling and hybridization. DNA was extracted from formalin-fixed, paraffin-embedded tumor tissue according to protocols from the UCSF Waldman Laboratory,
San Francisco, CA, USA (http://cc.ucsf.edu/people/waldman/ Protocols/paraffin.html), with an additional purification step using Phase Lock Gel tubes (Eppendorf AG, Hamburg, Germany). DNA quality was assessed using a Ready-ToGo RAPD analysis kit (GE Healthcare, Little Chalfont, UK) with primers 5'-AATCGGGCTG-3' and 5'-GAACGGGTG-3'. PCR products were validated on a Bioanalyzer (Agilent Technologies, Santa Clara, CA, USA). Tiling 32k BAC microarrays, with contiguous genome-wide coverage, were produced at the Microarray DNA Resource Centre, SCIBLU Genomics, Department of Oncology, Lund University, Sweden (http://www.lth.se/sciblu). Labeling and hybridization were performed as described by Jonsson et al (15). In short, 2-8 $\mu \mathrm{g}$ tumor DNA and $2 \mu \mathrm{g}$ reference DNA (Promega Corp., Madison, WI, USA) were labeled with Cy3-dCTP and Cy5-dCTP, using BioPrime Array CGH Genomic Labeling System (Invitrogen Life Technologies, Carlsbad, CA, USA). Tumor and reference DNA were pooled and mixed with Human COT-1 DNA (Invitrogen). Hybridizations were conducted using the MAUI Hybridization System (BioMicro systems Inc., Salt Lake City, UT, USA) and the slides were scanned in an Agilent Microarray Scanner (Agilent Technologies).

Image processing and data analysis. Image analysis was carried out using the GenePix ${ }^{\mathrm{TM}}$ Pro. 4.1 software (Axon Instruments Inc., Foster City, CA, USA) and quantified data matrices were uploaded in the web based BioArray Software Environment software (BASE) (16). Positive and nonsaturated spots were background corrected using the median background intensities for each channel and $\log _{2}$ ratios were calculated from the background corrected intensities. Data were filtered for flagged features and spots below signal to noise ratio 3 for both channels were eliminated. Data were normalized using an implementation of a population based LOWESS algorithm (17). Automatic breakpoint detection was performed using Circular Binary Segmentation (18). Copy number gains, losses and high level amplifications were determined by comparing segmented data to sample adaptive thresholds as described (17). The fraction of the genome altered was calculated as the total number of base pairs altered divided by the number of base pairs in the entire genome. Comparisons of data were made with regard to underlying mutation, histological type (serous vs. endometrioid, serous vs mucinous and endometrioid vs mucinous), grade (grade I and II vs. grade III and IV) and age at onset of disease ( $<40$ years vs. $>41$ years) using $\mathrm{MeV} 4.1 .01$ software (http://www.tm4.org/mev.html) on segmented, logged values for permutation analysis. Further, unsupervised hierarchical clustering through the Pearson correlation distance metric was used (19). By applying significant testing for aberrant copy number (STAC) analysis to the sporadic, BRCA1 and MMR associated subgroups we determined the statistical difference in copy number alterations (20). Regions with a frequency or footprint $\mathrm{p}$-value $<0.05$ were considered significantly altered. Copy number variants and altered telomeric and centromeric regions were excluded.

Microsatellite instability (MSI). MSI analysis was performed to ensure representative tumor DNA in the genetically stable 
Hierarchical cluster analysis

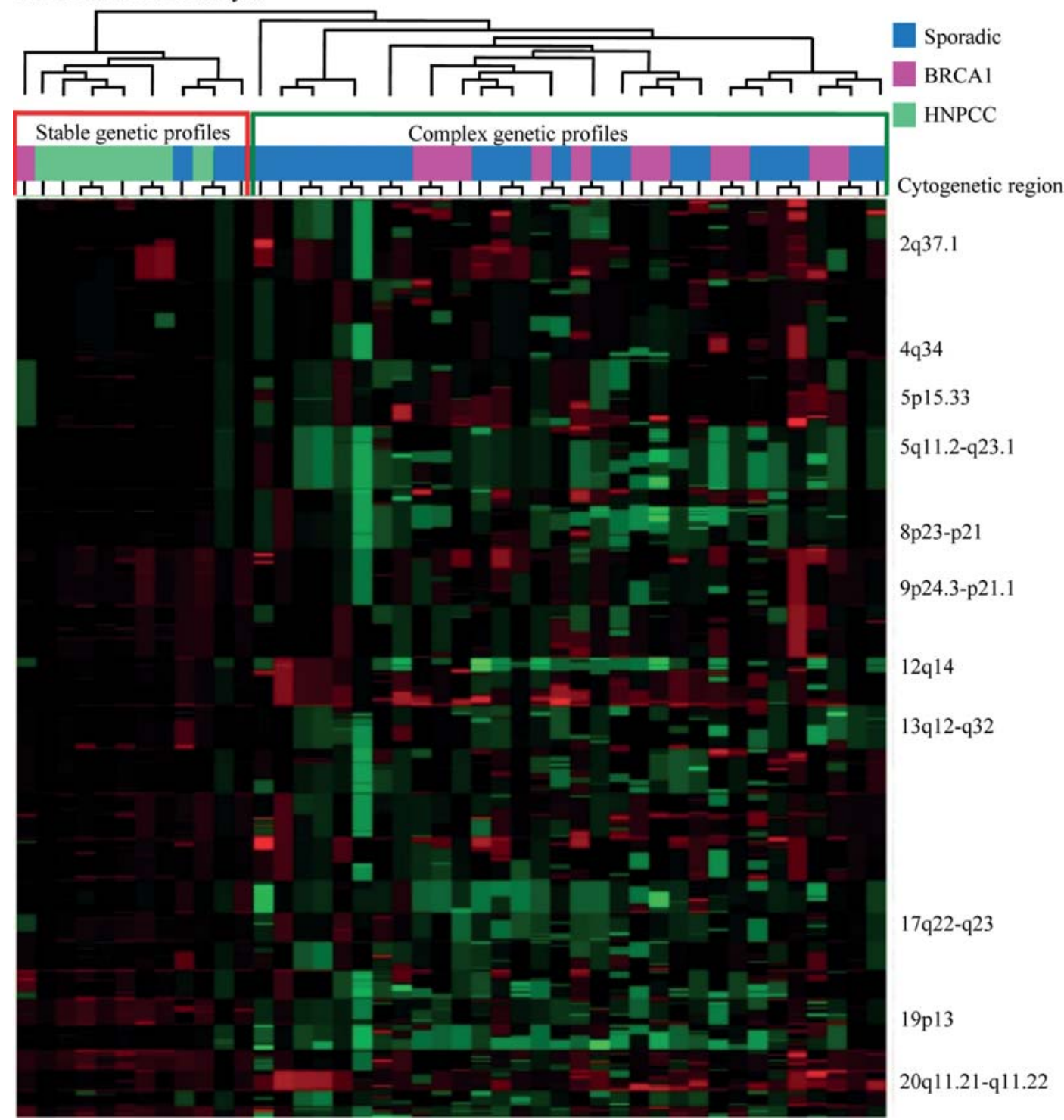

Figure 1. Unsupervised hierarchical cluster analysis of 12 BRCA1 mutated (pink), 8 MMR defective (green) and 24 sporadic (blue) ovarian tumors hybridized on 32k BAC arrays. Through cluster analysis two distinctive clusters related to genetic complexity were identified. One cluster consisted of tumors with complex genetic profiles with multiple gains and losses with an average of $41 \%$ of the genome altered, whereas the tumors of the other cluster had stable genetic profiles with an average of $18 \%$ of the genome altered. BAC clones are ordered according to their genomic position. Cytogenetic bands of regions of interest are labeled on the right.

HNPCC subset. DNA was extracted as described under 'DNA extraction, labeling and hybridization 'and MSI status was determined using the markers BAT25, BAT26, BAT34C4, BAT40, D2S123, D5S346, D17S787 and a microsatellite in the MSH6 gene. Primer sequences and analysis conditions are available from the authors upon request. The PCR products were analyzed on the ABI PRISM ${ }^{\text {TM }} 3130$ Genetic Analyzer (Applied Biosystems, Foster City, CA, USA). MSI was defined as the presence of additional peaks and was classified as MSI low if only one marker showed instability and as MSI high if at least two markers showed instability.

\section{Results}

DNA copy number alterations. Unsupervised hierarchical cluster analysis split the 44 ovarian cancers into two major clusters related to the degree of genetic complexity without relation to histology, age or tumor status (Fig. 1). Complex alterations with mean $41 \%$ of the genome altered were found in $21 / 24$ sporadic and $11 / 12$ BRCA1 tumors, whereas stable genetic profiles, with mean $18 \%$ of the genome altered were identified in all $8 \mathrm{HNPCC}$ tumors, in three of 24 sporadic tumors and in one of 12 BRCA1 tumors. The cluster analysis identified four outliers: three mucinous sporadic tumors and one serous BRCA1 tumor, which clustered among the tumors with stable genetic profiles. Gains on chromosomes 17 and 19 were commonly found in the genetically stable HNPCCassociated tumors and were present in 10 and 11 of the 12 tumors, respectively. MSI performed in the HNPCC associated tumors revealed MSI in 7/8 tumors with an MSI low pattern in five, and an MSI high phenotype in two.

Recurrent gains identified in at least $50 \%$ of BRCA1 and sporadic tumors included chromosomal regions 1q, 5p, 8q, $12 \mathrm{p}, 19 \mathrm{p}$ and 20 and losses on $4,5 \mathrm{q}, 8 \mathrm{p}, 9 \mathrm{q}, 14 \mathrm{q}$ and $17 \mathrm{p}$ 
Table II. Regions altered in $\geq 50 \%$ of the tumors.

\begin{tabular}{|c|c|c|c|c|c|c|}
\hline \multirow[b]{2}{*}{$\begin{array}{l}\text { Cytogenetic } \\
\text { location }\end{array}$} & \multirow[b]{2}{*}{$\begin{array}{l}\text { Start position } \\
\quad(\mathrm{Mb})\end{array}$} & \multirow[b]{2}{*}{$\begin{array}{l}\text { Size } \\
(\mathrm{Mb})\end{array}$} & \multicolumn{2}{|c|}{$\%$ of tumors with change } & \multirow[b]{2}{*}{$\begin{array}{l}\text { P-value } \\
\mathrm{p}<0.05\end{array}$} & \multirow[b]{2}{*}{ Candidate target genes } \\
\hline & & & $\overline{\text { Sporadic }}$ & $\overline{\mathrm{HBOC}}$ & & \\
\hline \multicolumn{7}{|l|}{$\begin{array}{l}\text { Copy number } \\
\text { gains } \geq 50 \%\end{array}$} \\
\hline $1 \mathrm{q} 21.2-\mathrm{q} 25.1$ & 146.8 & 23.8 & 53 & 23 & & $\begin{array}{l}\text { MUC1, UBE2Q1, RAB13, CREB3L4, } \\
\text { CREG1 }\end{array}$ \\
\hline $5 p 15.33$ & 0.000001 & 156.6 & 69 & 62 & & \\
\hline $6 \mathrm{p} 22.1-\mathrm{p} 21.1$ & 29.8 & 14.8 & 50 & 57 & & $T U B B, M D 1 L 3, V E G F$ \\
\hline $7 \mathrm{p} 22.3$ & 0.5 & 2 & 60 & 25 & & \\
\hline $7 q 36.3$ & 156.1 & 2.2 & 21 & 50 & & \\
\hline $8 q 24.12-q 24.3$ & 119.9 & 26.4 & 51 & 72 & & $M Y C$ \\
\hline $10 q 26.3$ & 134 & 1.2 & 52 & 42 & & \\
\hline $11 \mathrm{q} 13.2-\mathrm{q} 24.3$ & 65.8 & 6.6 & 34 & 50 & & $C C N D 1, F G F 4, F G F 3$, EMSY \\
\hline $12 \mathrm{p} 13.33-\mathrm{p} 13.31$ & 0.000001 & 8 & 68 & 60 & & $C D 4, K R A S$ \\
\hline $16 \mathrm{p} 13.3$ & 0.5 & 2.6 & 60 & 33 & & \\
\hline $17 \mathrm{p} 13.3$ & 0.7 & 0.4 & 20 & 50 & & \\
\hline $19 p 13.11$ & 17.6 & 1.6 & 54 & 43 & & $J A K 3, J U N D, R A B 3 A, E L L, M E C T 1, G D F 1$ \\
\hline 20p13 & 0.000001 & 3.1 & 74 & 51 & & $\begin{array}{l}\text { TRIB3, CSNK2A1, ANGPT4, CDC25B, } \\
\text { GNRH2, ADAM33 }\end{array}$ \\
\hline $20 q 11.21-q 13.33$ & 29.4 & 33.1 & 73 & 43 & & \\
\hline $20 q 11.21-q 11.22$ & 29.7 & 3.4 & 87 & 50 & 0.04 & $\begin{array}{l}\text { BCL2L1, PDRG1, RP11-49G10,8, E2F1, } \\
\text { PAK1, TGIF2, RBL1, GHRH, SRC, TGM2 }\end{array}$ \\
\hline $20 q 13.12$ & 45 & 5 & 66 & 58 & & \\
\hline $20 q 13.33$ & 59.7 & 2.8 & 87 & 88 & & \\
\hline \multicolumn{6}{|l|}{ Copy number } & \\
\hline 4p16-p14 & 10 & 27.3 & 29 & 50 & & \\
\hline $4 q 12-q 31.21$ & 53.2 & 93.6 & 46 & 63 & & $\begin{array}{l}\text { RASSF6, AREG, MAPK10, SCYE1, CASP6, } \\
\text { PRDM5, GAB1, SMAD1 }\end{array}$ \\
\hline $4 \mathrm{q} 22.1-\mathrm{q} 28.3$ & 89.7 & 42 & 54 & 72 & & \\
\hline $4 q 31.23-q 35.2$ & 148.9 & 42.2 & 47 & 69 & & \\
\hline $4 q 34.3-q 35.1$ & 182.2 & 1.8 & 52 & 83 & 0.005 & VEGFC,CASP3 \\
\hline $5 q 11.2-q 23.1$ & 53.8 & 63.5 & 41 & 74 & & $E R B B 2 I P, E N C 1, M S H 3, E D I L 3, R A S A 1$ \\
\hline 7p21.3-p21.1 & 8.1 & 11.4 & 21 & 55 & & \\
\hline $8 \mathrm{p} 23.3-\mathrm{p} 21.3$ & 0.2 & 21.2 & 47 & 95 & 0.002 & TUSC3, MTUS1, NAT1, NAT2 \\
\hline 8p21.3-p12 & 23.3 & 13.8 & 42 & 77 & 0.02 & $N R G 1$ \\
\hline $9 p 13.1-q 21.11$ & 38.8 & 30 & 36 & 53 & & \\
\hline $9 q 21.31-q 21.32$ & 79.2 & 3.8 & 52 & 42 & & TBCID2, TGFBRI \\
\hline $9 q 22.33-q 31.1$ & 99.5 & 1 & 36 & 50 & & \\
\hline 9q33.1-q33.2 & 115.8 & 4.4 & 52 & 24 & & \\
\hline $12 q 14.1$ & 56.9 & 3.4 & 4 & 50 & 0.01 & \\
\hline $12 \mathrm{q} 21.2-\mathrm{q} 23.1$ & 76.9 & 23 & 15 & 56 & 0.02 & \\
\hline 13q12.13-q32.2 & 25.1 & 72.1 & 35 & 72 & 0.03 & $\begin{array}{l}C D X 2, \text { FLT3, HSPH1, BRCA2, STARD13, } \\
L H F P, F O X O 1 A, L C P 1, R B 1\end{array}$ \\
\hline $14 q 12-q 21.3$ & 11 & 1.4 & 28 & 50 & & \\
\hline $14 \mathrm{q} 22.1-\mathrm{q} 23.2$ & 31.3 & 17.8 & 25 & 50 & & \\
\hline $14 q 24.3-q 32.11$ & 50.2 & 11.9 & 29 & 54 & & \\
\hline $14 q 32.33$ & 77.7 & 10.6 & 38 & 50 & & \\
\hline $14 q 32.33$ & 105.8 & 0.4 & 24 & 50 & & \\
\hline $15 q .11 .2$ & 18.3 & 4.7 & 32 & 57 & & \\
\hline $16 q 21-q 22.1$ & 57 & 7.8 & 47 & 65 & & \\
\hline $16 \mathrm{q} 22.1-\mathrm{q} 24.2$ & 65.5 & 21 & 38 & 60 & & $T R A D D, M M P 2, E 2 F, A D A M T S 18$ \\
\hline $17 \mathrm{p} 13.1-\mathrm{q} 21.2$ & 8.7 & 28.1 & 30 & 65 & & MAX,OVCA2, HICl \\
\hline
\end{tabular}


Table II. Continued.

\begin{tabular}{|c|c|c|c|c|c|c|}
\hline \multirow[b]{2}{*}{$\begin{array}{l}\text { Cytogenetic } \\
\text { location }\end{array}$} & \multirow[b]{2}{*}{$\begin{array}{l}\text { Start position } \\
\quad(\mathrm{Mb})\end{array}$} & \multirow[b]{2}{*}{$\begin{array}{l}\text { Size } \\
(\mathrm{Mb})\end{array}$} & \multicolumn{3}{|c|}{$\%$ of tumors with change } & \multirow[b]{2}{*}{ Candidate target genes } \\
\hline & & & $\overline{\text { Sporadic }}$ & $\overline{\mathrm{HBOC}}$ & $\begin{array}{r}\text { P-value } \\
\mathrm{p}<0.05\end{array}$ & \\
\hline $17 \mathrm{p} 12$ & 10.9 & 4 & 67 & 73 & & \\
\hline $17 \mathrm{p} 11.2$ & 21.1 & 0.5 & 55 & 67 & & \\
\hline $17 q 22.1-q 23.2$ & 40.9 & 14.2 & 25 & 59 & 0.03 & \\
\hline 18p11.32-p11.21 & 0.2 & 11.6 & 27 & 51 & & $\begin{array}{l}B C L 2, M A L T 1, S M A D 4, S M A D 2, S M A D 7, \\
S S 18, D C C, C D H 7\end{array}$ \\
\hline $18 p 11.21$ & 14.1 & 0.9 & 11 & 61 & 0.003 & \\
\hline $18 q 11.2-q 23$ & 17.3 & 58.9 & 53 & 68 & & \\
\hline $18 \mathrm{q} 12.3$ & 37.1 & 0.6 & 52 & 75 & & \\
\hline $18 q 21.1-q 23$ & 45.8 & 30 & 69 & 74 & & BIRC7, CTSZ \\
\hline $19 p 13.3$ & 5 & 0.1 & 8 & 50 & 0.0003 & \\
\hline $19 \mathrm{p} 13.2$ & 8.7 & 0.2 & 12 & 50 & 0.04 & \\
\hline 21p13-q21.1 & 0.000001 & 22.4 & 34 & 52 & & МYOB $18 B$ \\
\hline $22 q 13.31-q 13.33$ & 4.3 & 56 & 37 & 51 & & GTSE1 \\
\hline
\end{tabular}

aDetermined using student's t-test.

A Whole genome frequency plot

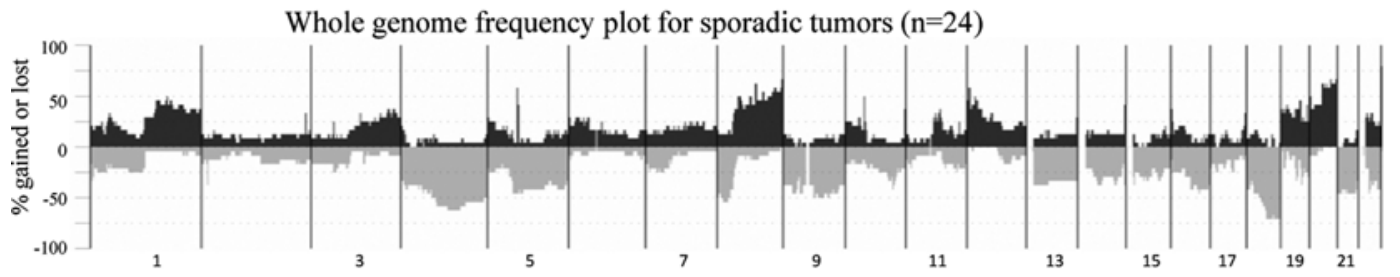

B

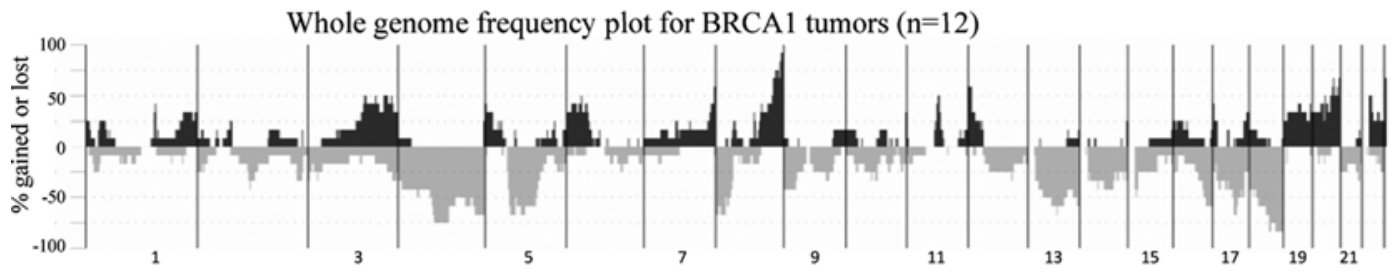

C

Whole genome frequency plot for MMR defective tumors $(n=8)$

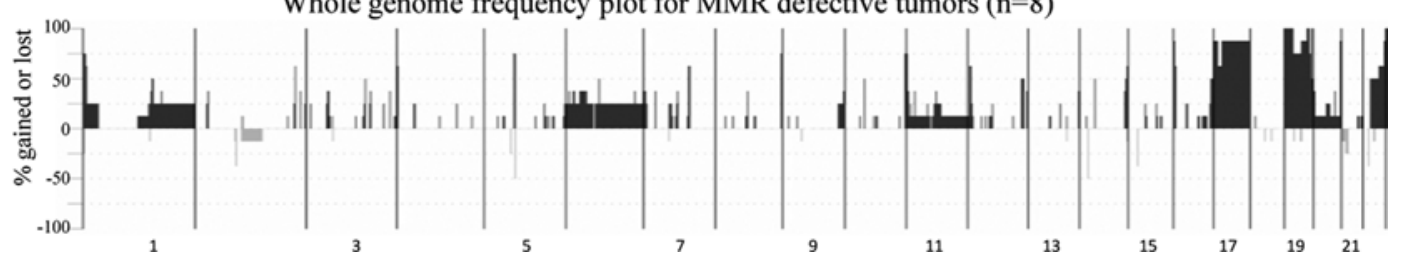

Figure 2. The frequency plot summarizes gains and losses in ovarian cancers from (A) 24 sporadic tumors, (B) 12 BRCA1 associated tumors and (C) 8 MMR defective tumors.

(Table II). Gains were more predominant in the sporadic tumors, whereas losses were more common in the BRCA1 tumors. When gains and losses present in $\geq 50 \%$ of the tumors were considered, losses of 4q34, 8p23-p21, 8p21-p12, 12q14.1, 12q21-q23, 13q12-q32, 17q22.1-q23.2, 18p11-p21, $19 \mathrm{p} 13.3$ and $19 \mathrm{p} 13.2$ were significantly over-represented in the BRCA1 tumors $(\mathrm{p}<0.05)$ (Table II). Potential discriminating genes within these regions include $B R C A 2$, FOXO1A and RB1. Gains of 20q11.21-q11.22 were significantly more common in the sporadic tumors and harbor genes such as $S R C, E 2 F 1$ and $P A K 1$. BRCAI promoter methylation pyrosequencing demonstrated promoter methylation in two tumors, both of which clustered in the genetically complex group (Fig. 1) (data not shown). 
Table III. High level amplifications and homozygous deletions of genetically stable and complex ovarian tumors.

\begin{tabular}{|c|c|c|c|c|c|c|}
\hline $\begin{array}{l}\text { Tumor } \\
\text { subset }\end{array}$ & $\begin{array}{l}\text { Cytogenetic } \\
\text { location }\end{array}$ & $\begin{array}{l}\text { Start position } \\
\qquad(\mathrm{Mb})\end{array}$ & $\begin{array}{l}\text { Size } \\
(\mathrm{Mb})\end{array}$ & $\begin{array}{l}\text { Candidate } \\
\text { target genes }\end{array}$ & Genetic profile & Histology \\
\hline \multicolumn{7}{|c|}{$\begin{array}{l}\text { High level } \\
\text { amplifications }\end{array}$} \\
\hline Sporadic & 1p34.3-p34.2 & 36.4 & 7.0 & & Complex & Endometrioid \\
\hline Sporadic & $1 \mathrm{p} 32.3$ & 54.9 & 0.2 & & Complex & Serous \\
\hline Sporadic & 2p14-p13.3 & 67.9 & 1.1 & $A P L F, P R O K R I$ & Complex & Serous \\
\hline Sporadic & $2 \mathrm{p} 11.2$ & 84.4 & 1.0 & TMSB10, TCF7L1 & Complex & Serous \\
\hline Sporadic & 3p12.2-p12.1 & 81.7 & 2.3 & & Complex & Endometrioid \\
\hline HBOC & $3 q 26.1-q 26.31$ & 168.7 & 7.0 & TERC, TNFSF10, EIF5A2 & Complex & Serous. endometrioid \\
\hline Sporadic & $3 q 27.1-\mathrm{p} 27.2$ & 185.3 & 0.8 & & Complex & Serous \\
\hline Sporadic & $4 p 16.3$ & 0.01 & 2.7 & $\begin{array}{l}F G F R L 1, C T B P 1, T A C C 3, \\
F G F R 3, W H S C 1, W H S C 2\end{array}$ & Complex & Serous \\
\hline Sporadic & $4 q 13.3$ & 73.9 & 1.1 & $A F P, I L-8$ & Complex & Serous \\
\hline HBOC & 5p15.33-p15.1 & 0.1 & 18.3 & TERT, ADAMTS16 & Complex & Serous \\
\hline Sporadic & 5p13.3-p13.2 & 33.0 & 4.5 & RAD1, SKP2 & Complex & Serous \\
\hline Sporadic & $6 \mathrm{p} 22.3$ & 18.7 & 4.8 & $E 2 F 3$ & Complex & Serous \\
\hline HBOC & $6 \mathrm{p} 21.32-\mathrm{p} 21.2$ & 33.4 & 3.8 & $\begin{array}{l}\text { HMGA1, PPARD, } \\
\text { CDKN1A, PIM1 }\end{array}$ & Complex & Endometrioid \\
\hline Sporadic & $6 \mathrm{p} 21.2-\mathrm{p} 21.1$ & 37.6 & 6.2 & $C C N D 3$ & Complex & Serous \\
\hline Sporadic & 8p21.2-p21.1 & 26.7 & 1.4 & & Complex & Serous \\
\hline $\begin{array}{l}\text { HBOC. } \\
\text { sporadic }\end{array}$ & 8p12-p11.23 & 36.7 & 1.9 & FGFRI & Complex & Serous \\
\hline HBOC & $8 p 11.21$ & 41.8 & 0.9 & $I K B K B, P O L B$ & Complex & Serous \\
\hline $\begin{array}{l}\text { HBOC. } \\
\text { sporadic }\end{array}$ & $8 \mathrm{q} 24.21$ & 127.8 & 3.2 & $M Y C, P V T 1$ & Complex & Serous \\
\hline Sporadic & $10 p 11.22$ & 32.5 & 1.6 & $N R P 1$ & Complex & Serous \\
\hline Sporadic & $10 \mathrm{q} 21.1-\mathrm{q} 21.3$ & 59.9 & 5.2 & & Complex & Serous \\
\hline $\begin{array}{l}\text { HBOC. } \\
\text { sporadic }\end{array}$ & $11 \mathrm{q} 13.4-\mathrm{q} 13.5$ & 74.0 & 1.2 & $E M S Y$ & Complex & Serous \\
\hline Sporadic & $11 q 14.1$ & 77.7 & 2.6 & & Complex & Serous \\
\hline Sporadic & $11 \mathrm{q} 22.1-\mathrm{q} 22.2$ & 99.4 & 2.4 & $P G R$ & Complex & Serous \\
\hline Sporadic & $11 \mathrm{q} 22.2$ & 101.8 & 0.5 & $\begin{array}{l}\text { Matrix metalloproteinase } \\
(M M P) \text { family cluster }\end{array}$ & Complex & Serous \\
\hline Sporadic & $13 q 22.3-q 31.1$ & 76.9 & 1.2 & & Complex & Serous \\
\hline HBOC & $14 q 13.1-q 13.3$ & 33.8 & 2.8 & & Complex & Endometrioid \\
\hline Sporadic & $15 q 22.2$ & 58.4 & 1.7 & $A N X A 2$ & Complex & Serous \\
\hline Sporadic & $16 \mathrm{p} 11.2$ & 29.5 & 2.0 & & Complex & Serous \\
\hline Sporadic & $17 \mathrm{p} 13.1$ & 7.0 & 2.1 & TP53 & Complex & Serous \\
\hline Sporadic & $17 q 12-q 21.1$ & 35.0 & 0.8 & $E R B B 2$ & Complex. stable & Serous. mucinous \\
\hline Sporadic & $18 \mathrm{q} 11.2$ & 18.0 & 2.1 & RBBP8, cables 1 & Complex & Serous \\
\hline Sporadic & $18 \mathrm{q} 21.1$ & 44.0 & 0.9 & & Stable & Mucinous \\
\hline Sporadic & $18 q 21.33$ & 58.1 & 0.7 & $B C L 2$ & Stable & Mucinous \\
\hline Sporadic & $18 \mathrm{q} 22$ & 73.3 & 0.4 & & Stable & Mucinous \\
\hline Sporadic & 19p13.13-p13.12 & 13.7 & 0.7 & & Complex & Serous. endometrioid \\
\hline Sporadic & $19 q 12$ & 33.7 & 1.4 & CCNE1 & Complex & Serous \\
\hline Sporadic & $19 q 13.32$ & 50.6 & 1.1 & $F O S B, H I F 3 A$ & Complex & Serous \\
\hline HBOC & $20 \mathrm{p} 13$ & 0.03 & 4.6 & $C D C 25 B$ & Complex & Endometrioid \\
\hline $\begin{array}{l}\text { HBOC. } \\
\text { sporadic }\end{array}$ & 20q13.12-q13.13 & 43.1 & 3.4 & WFDC2, MMP9, & Complex & Serous \\
\hline Sporadic & $20 q 13.13$ & 47.1 & 2.0 & CSE1L, PTPN1, ADNP & Complex & Serous \\
\hline HBOC & $20 q 13.33$ & 60.0 & 0.3 & CDH4, BIRC7 & Complex & Endometrioid \\
\hline Sporadic & $22 q 12.3$ & 30.5 & 1.6 & & Complex & Serous \\
\hline
\end{tabular}


Table III. Continued.

\begin{tabular}{|c|c|c|c|c|c|c|}
\hline $\begin{array}{l}\text { Tumor } \\
\text { subset }\end{array}$ & $\begin{array}{c}\text { Cytogenetic } \\
\text { location }\end{array}$ & $\begin{array}{l}\text { Start position } \\
(\mathrm{Mb})\end{array}$ & $\begin{array}{l}\text { Size } \\
(\mathrm{Mb})\end{array}$ & $\begin{array}{c}\text { Candidate } \\
\text { target genes }\end{array}$ & Genetic profile & Histology \\
\hline \multicolumn{7}{|c|}{$\begin{array}{l}\text { Homozygous } \\
\text { deletions }\end{array}$} \\
\hline HBOC & $4 q 35.2$ & 187.9 & 0.2 & $F A T 1$ & Complex & Serous \\
\hline HBOC & $5 q 12.3-q 13.1$ & 66.3 & 1.4 & & Complex & Serous \\
\hline HBOC & $9 \mathrm{p} 21.3-\mathrm{p} 21.1$ & 21.5 & 0.6 & $C D K N 2 A, C D K N 2 B$ & Complex & Serous \\
\hline HBOC & $10 q 23.31$ & 90.0 & 0.4 & PTEN & Complex & Endometrioid \\
\hline Sporadic & $14 \mathrm{q} 31.3$ & 83.7 & 2.2 & & Complex & Serous \\
\hline
\end{tabular}

A

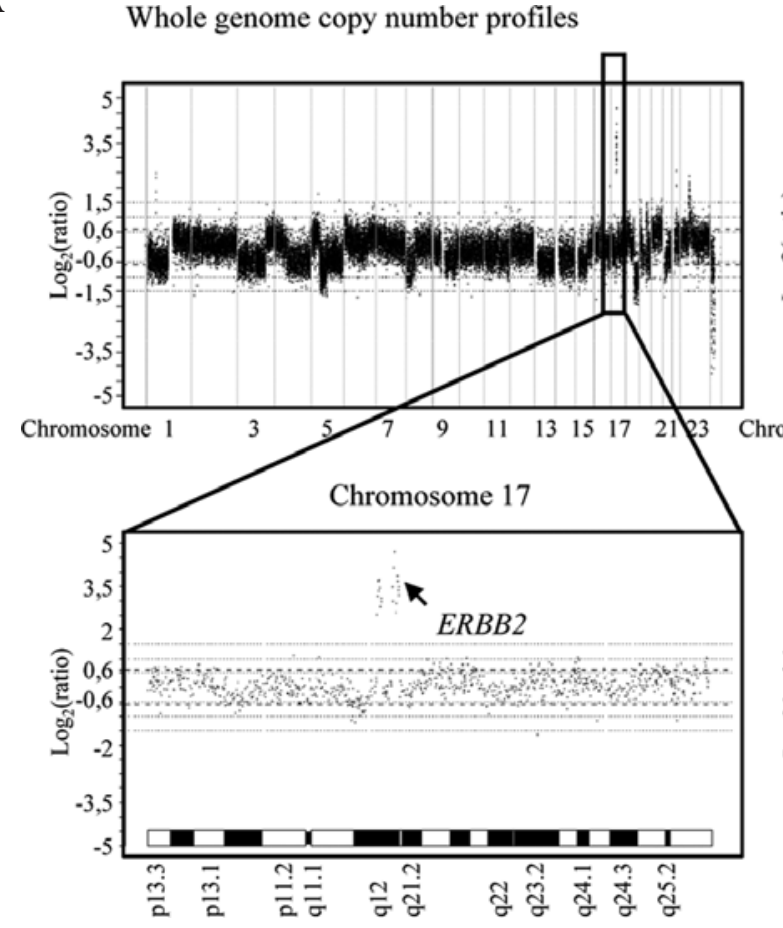

B

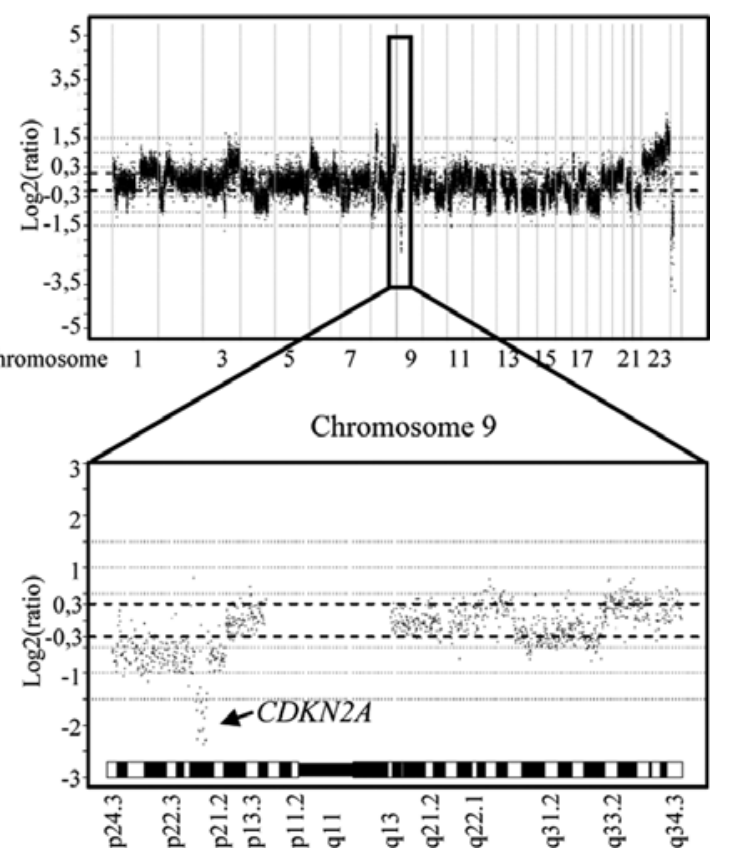

Figure 3. Pictured are two ovarian cancers demonstrating multiple gains and losses. (A) Characteristic alterations for sporadic tumors include gains on 1q, 5p and 20q and losses on 4q and 18q. Zoom-in on chromosome 17 reveals a high level amplification on 17q12, harboring the ERBB2 gene. (B) Characteristic alterations for BRCA1 tumors include gains on $6 \mathrm{p}$ and $8 \mathrm{q}$ and losses on $4 \mathrm{q}, 5 \mathrm{q}$ and $8 \mathrm{p}$. Zoom-in on chromosome 9 reveals a probable homozygous deletion on 9p21.3, harboring the CDKN2A gene.

High level amplifications and homozygous deletions. High level amplifications (segments with $\log _{2}$ ratios $>5$ times the adaptive threshold) that affected 42 different loci were observed in 18 tumors. High level amplifications were as common in the sporadic tumors $(12 / 24)$ as in the BRCA1 tumors (7/12) and were not observed in any HNPCC associated tumors (Table III). Recurrent high level amplification involved the ERBB2 locus on $17 \mathrm{q} 12$ in two sporadic tumors, EMSY (11q13.5) in three tumors (one BRCA1 and two sporadic) and $M Y C(8 q 24.21)$ in one BRCA1 and one sporadic tumor. Six tumors (two sporadic and four BRCA1 tumors) showed putative homozygous deletions (segments with $\log _{2}$ ratios $<7$ times the threshold) in 9 loci including 4q35 (FAT), 9p21.3-p21.1 (CDKN2A,CDKN2B) in two tumors and 10q23.31 (PTEN) (Table III, Fig. 3). Significantly altered regions identified by STAC are listed in Table IV.

\section{Discussion}

The multitude of copy number changes demonstrated in the sporadic tumor subset included several alterations previously linked to ovarian cancer, e.g. gains at 1q, 3q (PIK3CA), 8q $(M Y C), 11 \mathrm{q}(P A K 1, C C N D 1, F G F 4 / F G F 3), 12 \mathrm{p}(K R A S 2)$, 17q (ERBB2), 20q (STK15) and recurrent losses of 4q, 9p (CDKN2A), 13q (BRCA2) and 16q (Table II) (21-23). 
Table IV. Significant testing for aberrant copy number analysis.

\begin{tabular}{|c|c|c|c|c|}
\hline $\begin{array}{l}\text { Tumor } \\
\text { subset }\end{array}$ & $\begin{array}{l}\text { Cytogenetic } \\
\text { location }\end{array}$ & $\begin{array}{l}\text { Start position } \\
\qquad(\mathrm{Mb})\end{array}$ & $\begin{array}{l}\text { Size } \\
(\mathrm{Mb})\end{array}$ & Candidate target genes \\
\hline \multicolumn{5}{|c|}{$\begin{array}{l}\text { Copy number } \\
\text { gains }\end{array}$} \\
\hline HBOC & $1 \mathrm{q} 21.3-\mathrm{q} 22$ & 151.1 & 2.2 & MUC1, UBE2Q1, RAB13, CREB3L4, CREG1 \\
\hline $\mathrm{HBOC}$ & $5 q 35.2-q 35.3$ & 175.1 & 5.7 & NSD1, MAPK9, GNB2L1 \\
\hline $\mathrm{HBOC}$ & $8 q 24.22$ & 132.8 & 3 & \\
\hline HBOC & $8 q 24.3$ & 140.5 & 5.5 & PSCA, MAFA, BOP1, RECQLA \\
\hline $\mathrm{HBOC}$ & $9 q 31.3-q 34.3$ & 111.7 & 26.3 & $\begin{array}{l}\text { TNC, DAB2IP, SET, PPP2R4, PRRX2, FNBP1, ABL1, } \\
\text { NUP214, TSC1, NOTCH1 }\end{array}$ \\
\hline $\mathrm{HBOC}$ & $11 \mathrm{q} 13.2-\mathrm{q} 13.4$ & 65.6 & 5.3 & $C C N D 1, F G F 4, F G F 3$ \\
\hline HBOC & $12 \mathrm{p} 13.33-\mathrm{p} 13.31$ & 0.000001 & 6.9 & CCND2 \\
\hline $\mathrm{HBOC}$ & $17 \mathrm{p} 13.3-\mathrm{p} 13.1$ & 0.00001 & 7.9 & TP53 \\
\hline HBOC & $17 \mathrm{q} 24.3-\mathrm{q} 25.3$ & 67.9 & 10.8 & $\begin{array}{l}\text { GRB2, RECQL5, ST6GALNAC1, SEPT9, ENPP7, } \\
\text { ASPSCR1, RAC3 }\end{array}$ \\
\hline HBOC & $20 q 13.33$ & 61.2 & 1.2 & \\
\hline HBOC & $22 q 11.21-q 11.22$ & 16.3 & 4.6 & CLTCL1, sept52L1, PPIL2, \\
\hline HNPCC & $2 \mathrm{p} 23.3$ & 27.3 & 0.4 & \\
\hline HNPCC & $2 q 35$ & 219.9 & 0.4 & \\
\hline HNPCC & $3 \mathrm{p} 21.31$ & 49.5 & 0.9 & MST1R, RBM5, SEMA3F, HYAL1, HYAL2, RASSF1 \\
\hline HNPCC & $3 q 21.3$ & 130.2 & 0.4 & \\
\hline HNPCC & $4 \mathrm{p} 16.3$ & 1.0 & 1.9 & CTBP1, TACC3, FGFR3, WHSC1, WSC2 \\
\hline HNPCC & $6 q 13$ & 74.0 & 0.5 & $D D X 43$ \\
\hline HNPCC & $7 q 22.1$ & 99.5 & 1.5 & \\
\hline HNPCC & $11 \mathrm{p} 15.5-\mathrm{p} 15.4$ & 0.000001 & 3.9 & HRAS, CARS, NUP98 \\
\hline HNPCC & $12 \mathrm{p} 13.31$ & 6.2 & 1.9 & ZNF384 \\
\hline Sporadic & $2 q 37.1$ & 232.2 & 1 & \\
\hline Sporadic & $3 \mathrm{p} 21.31$ & 50.4 & 0.3 & \\
\hline Sporadic & $4 \mathrm{p} 16.3-\mathrm{p} 16.1$ & 0.1 & 8.4 & FGRGL1, CTBP1, TACC3, FGFR3, WHSC1, WHSC2 \\
\hline Sporadic & $5 q 13.2$ & 68.5 & 1 & $C C N B 1$ \\
\hline Sporadic & 8p11.22-p11.1 & 39.8 & 5.3 & $I K B K B, P O L B$ \\
\hline Sporadic & $9 \mathrm{q} 34.11-\mathrm{q} 34.3$ & 127.7 & 10.3 & $\begin{array}{l}\text { SET, PPP2R4, PRRX2, FNBP1, ABL1, NUP } 214, \\
\text { TCS1, NOTCH1 }\end{array}$ \\
\hline Sporadic & $10 p 11.22$ & 31.8 & 1 & \\
\hline Sporadic & $10 q 11.21$ & 42.9 & 1.7 & $R E T$ \\
\hline Sporadic & $11 \mathrm{q} 13.5$ & 75.8 & 0.8 & $E M S Y$ \\
\hline Sporadic & $12 \mathrm{p} 13.33-\mathrm{p} 13.31$ & 0.000001 & 6.9 & CCND2 \\
\hline Sporadic & $13 q 14.11$ & 43.5 & 0.5 & \\
\hline Sporadic & $13 q 14.2$ & 49.1 & 0.5 & \\
\hline Sporadic & $16 q 11.2$ & 40.7 & 4.4 & \\
\hline Sporadic & $16 q 23.1$ & 76.1 & 1 & \\
\hline Sporadic & $17 q 25.3$ & 76.7 & 2 & ASPSCR1, RAC3 \\
\hline Sporadic & $20 q 13.33$ & 61.3 & 0.8 & \\
\hline Sporadic & $21 \mathrm{q} 22.3$, end & 46.6 & 0.3 & $S 100 B$ \\
\hline \multicolumn{5}{|c|}{$\begin{array}{l}\text { Copy number } \\
\text { losses }\end{array}$} \\
\hline HBOC & $3 q 27.2-q 29$ & 187.2 & 7.3 & ETV5, EIF4A2, BCL6, LPP \\
\hline HBOC & $5 q 11.2-q 13.2$ & 55.1 & 13.3 & $E R B B 2 I P, E N C 1$ \\
\hline HBOC & $8 \mathrm{p} 23.3-\mathrm{p} 21.3$ & 0.5 & 19.6 & TUSC3, MTUS1, NAT1, NAT2, GATA4 \\
\hline HBOC & $9 \mathrm{p} 24.3-\mathrm{p} 21.1$ & 0.4 & 27.7 & $\begin{array}{l}J A K 2, J M J D 2 C, P T P R D, P S I P 1, S H 3 G L 2, M L L T 3, \\
C D K N 2 A, C D K N 2 B\end{array}$ \\
\hline $\mathrm{HBOC}$ & $9 \mathrm{q} 22.32-\mathrm{q} 31.1$ & 95.8 & 4.8 & \\
\hline HBOC & $16 q 23.1-q 24.3$ & 74.4 & 14.4 & FANCA \\
\hline
\end{tabular}


Table IV. Continued.

\begin{tabular}{|c|c|c|c|c|}
\hline $\begin{array}{l}\text { Tumor } \\
\text { subset }\end{array}$ & $\begin{array}{l}\text { Cytogenetic } \\
\text { location }\end{array}$ & $\begin{array}{l}\text { Start position } \\
\quad(\mathrm{Mb})\end{array}$ & $\begin{array}{l}\text { Size } \\
(\mathrm{Mb})\end{array}$ & Candidate target genes \\
\hline \multicolumn{5}{|c|}{$\begin{array}{l}\text { Copy number } \\
\text { losses }\end{array}$} \\
\hline HBOC & $19 \mathrm{p} 13.2$ & 8.7 & 1 & \multirow{6}{*}{$E R B B 2 I P, E N C 1$} \\
\hline Sporadic & 1p36.32-p36.31 & 4.0 & 1.6 & \\
\hline Sporadic & $5 q 11.2-q 13.2$ & 51.7 & 16.7 & \\
\hline Sporadic & $8 \mathrm{p} 23.2$ & 2.7 & 0.1 & \\
\hline Sporadic & $8 \mathrm{p} 23.1-\mathrm{p} 21.3$ & 9.9 & 10.2 & \\
\hline Sporadic & $10 \mathrm{q} 25.1$ & 106.3 & 1.3 & \\
\hline Sporadic & $13 q 12.11-q 14.2$ & 18.4 & 30.7 & \multirow[t]{3}{*}{$\begin{array}{l}\text { CDX2, FLT3, HSPH1, BRCA2, STARD13, LHFP, } \\
\text { FOXO1A, LCP1, RB1 }\end{array}$} \\
\hline Sporadic & $14 q 32.33$ & 105.3 & 1 & \\
\hline Sporadic & $17 \mathrm{q} 22$ & 49.0 & 1.4 & \\
\hline
\end{tabular}

Data on BRCA1 ovarian cancer profiles suggest genetic complexity with gains of $2 \mathrm{q}, 3 \mathrm{q}, 6 \mathrm{p}, 7 \mathrm{q}, 8 \mathrm{q}, 20 \mathrm{q}$, and losses of $4 \mathrm{q}, 5 \mathrm{q}, 6 \mathrm{q}, 13 \mathrm{q}, 17 \mathrm{q}, 18 \mathrm{q}, 19,20 \mathrm{q}$, which is supported also by our findings $(24,25)$. Similarities have also been recognized between $\mathrm{HBOC}$ associated and sporadic breast cancer, e.g. with losses of $4 q$ and $5 q$ in both tumor types and BRCA1 inactivation is thus linked to genetically complex tumors without yet identified differences from the sporadic ovarian cancers (26-29). All eight HNPCC associated tumors were genetically stable. Recurrent gains affected chromosomes 17 and 19, though these regions are too large to allow distinction of potential target genes (Table II). The stable genetic profiles demonstrated in the HNPCC associated ovarian cancers are in line with the findings in colorectal cancer, in which tumors with germline MMR gene mutations or $M L H 1$ promotor methylation display near diploid genetic profiles with few structural changes (30-32).

STAC analysis was applied to the different tumor subsets in order to identify target genes within the regions specifically gained or lost (Table IV). A region on $17 \mathrm{p} 13$ harboring the TP53 gene was gained in a significant number of BRCA1 tumors. While point mutations is the most common mechanism for TP53 inactivation in ovarian cancer, amplifications has been reported in endometrioid cancers, which underscores that different genetic mechanisms apply in the development and progression of the various types of ovarian cancer $(2,33)$. Further, a number of oncogenes commonly gained in ovarian cancer such as HRAS and EMSY were identified. Mutations of EMSY are associated with poor outcome and serous histology. Candidate genes involved in tumor suppression included BRCA2 in the sporadic subset, GATA4 and FANCA in the BRCA1 tumors (34). Loss of FANCA proteins implicates that inhibition of the DNA damage signalling poly (ADP-ribose) polymerase (PARP) protein may be useful in a subset of sporadic tumors (35).

High level amplifications were predominantly found in sporadic and $B R C A 1$ ovarian cancers and included the potential target genes $P G R, C D C N 20, S M A D 7, T I M P 3$, $P A K 1$ and ERBB2 (Table III, Fig. 3). The ERBB2 locus at
$17 q 12$ plays a critical role in tumor cell proliferation with overexpression in up to three quarters of ovarian cancer (36). Frequent high level amplifications occurred in both sporadic and BRCA1 mutated tumors, which suggests that copy number dependent oncogene amplifications are central in these tumor subsets. Two BRCA1 tumors showed homozygous deletions of the $C D K N 2 A$ locus, which has been observed in 11-27\% of ovarian cancers, and has been linked to advanced stage and poor prognosis (Table III, Fig. 3) $(37,38)$. Lower degree of oncogene activation in MMR defective ovarian cancers compared to the BRCAl mutated and sporadic cases may have therapeutic consequences for the response to chemotherapeutic regimens and targeted therapies. BRCAl mutated ovarian cancers are generally resistant to paclitaxel, but responsive to platinum based drugs, whereas in vitro data suggest that MMR defective cells may be resistant to platinum based chemotherapy (39-41). Inhibition of $E R B B 2$ and $M Y C$ has been suggested to be less effective in hereditary ovarian cancers, whereas PARP inhibition and anti-angiogenic agents show promising results in HBOC associated ovarian cancers $(35,36,42)$.

The distinct genetic profiles recognized in the two major types of hereditary ovarian cancer reflect tumor development along different pathways with genetic complexity in the HBOC associated BRCAI mutated tumors and stable genetic profiles in the HNPCC associated MMR defective tumors. This is in concordance with the predominance of serous tumors in HBOC and endometrioid, clear cell, and mucinous tumors commonly diagnosed in HNPCC. A dualistic model for the development of ovarian cancer has been suggested with high grade serous and undifferentiated tumors developing de novo, whereas low grade serous carcinomas, mucinous, clear cell and low grade endometrioid tumors develop in a stepwise sequence (43). Cellular origin may differ with $\mathrm{HBOC}$ associated ovarian cancers suggested to originate in the fallopian tube secretory epithelium rather than the surface epithelium $(44,45)$. Different genetic pathways drive tumor development with mutations in TP53, PI3K/ $A K T$ pathway genes and $B R C A 1 / B R C A 2$ in the genetically 
unstable cases and mutations in KRAS, BRAF, PTEN and $\beta$ catenin frequently identified in the genetically stable ovarian cancers $(2,43,46,47)$. The differences in genetic complexity identified may be applied for refined diagnostics of HBOC and HNPCC associated ovarian cancer, and may also have implications for the response to chemotherapy and targeted therapies for individuals with hereditary ovarian cancer.

\section{Acknowledgements}

Financial support was granted from the Wallenberg foundation, the Swedish Cancer Society, the Swedish Research Council, the Nilsson Cancer Fund and the Region Skåne Research Funds. We would like to thank Dr Henrik Grönvall at the Umeå university for supplying material for the study.

\section{References}

1. Bewtra C, Watson P, Conway T, Read-Hippee C and Lynch HT: Hereditary ovarian cancer: a clinicopathological study. Int J Gynecol Pathol 11: 180-187, 1992.

2. Lynch HT, Casey MJ, Snyder CL, et al: Hereditary ovarian carcinoma: heterogeneity, molecular genetics, pathology, and management. Mol Oncol 3: 97-137, 2009.

3. Whittemore AS: Characteristics relating to ovarian cancer risk: implications for prevention and detection. Gynecol Oncol 55: S15-S19, 1994

4. Risch HA, McLaughlin JR, Cole DE, et al: Population brca1 and BRCA2 mutation frequencies and cancer penetrances: kin-cohort study in Ontario, Canada. J Natl Cancer Inst 98: 16941706,2006

5. Rubin SC, Benjamin I, Behbakht K, et al: Clinical and pathological features of ovarian cancer in women with germ-line mutations of BRCA1. N Engl J Med 335: 1413-1416, 1996.

6. Pal T, Permuth-Wey J, Kumar A and Sellers TA: Systematic review and meta-analysis of ovarian cancers: estimation of micro- satellite-high frequency and characterization of mismatch repair deficient tumor histology. Clin Cancer Res 14: 6847-6854, 2008.

7. Watson P, Butzow R, Lynch HT, et al: The clinical features of ovarian cancer in hereditary non-polyposis colorectal cancer. Gynecol Oncol 82: 223-228, 2001.

8. Moynahan ME, Cui TY and Jasin M: Homology-directed DNA repair, mitomycin-C resistance, and chromosome stability is restored with correction of a BRCA1 mutation. Cancer Res 61: 4842-4850, 2001.

9. Bell DA: Origins and molecular pathology of ovarian cancer Mod Pathol 18 (Suppl. 2): S19-S32, 2005.

10. Aarnio M, Sankila R, Pukkala E, et al: Cancer risk in mutation carriers of DNA-mismatch-repair genes. Int J Cancer 81: 214-218, 1999

11. Moller P, Hagen AI, Apold J, et al: Genetic epidemiology of BRCA mutations, family history detects less than $50 \%$ of the mutation carriers. Eur J Cancer 43: 1713-1717, 2007.

12. Boyd J, Sonoda Y, Federici MG, et al: Clinicopathologic features of BRCA-linked and sporadic ovarian cancer. JAMA 283: 2260-2265, 2000.

13. Malander S, Ridderheim M, Masback A, et al: One in 10 ovarian cancer patients carry germ line BRCA1 or BRCA2 mutations: results of a prospective study in southern Sweden. Eur J Cancer 40: 422-428, 2004.

14. Malander S, Rambech E, Kristoffersson U, et al: The contribution of the hereditary non-polyposis colorectal cancer syndrome to the development of ovarian cancer. Gynecol Oncol 101: 238-243,2006.

15. Jonsson G, Staaf J, Olsson E, et al: High-resolution genomic profiles of breast cancer cell lines assessed by tiling BAC array comparative genomic hybridization. Genes Chromosomes Cancer 46: 543-558, 2007.

16. Saal LH, Troein C, Vallon-Christersson J, Gruvberger S, Borg A and Peterson C: Bioarray software environment (base): a platform for comprehensive management and analysis of microarray data. Genome Biol 3: Software0003, 2002.
17. Staaf J, Jonsson G, Ringner M and Vallon-Christersson J: Normalization of array-CGH data: influence of copy number imbalances. BMC Genomics 8: 382, 2007.

18. Olshen AB, Venkatraman ES, Lucito R and Wigler M: Circular binary segmentation for the analysis of array-based DNA copy number data. Biostatistics 5: 557-572, 2004.

19. Saeed AI, Sharov V, White J, et al: TM4: a free, open-source system for microarray data management and analysis. Biotechniques 34: 374-378, 2003.

20. Diskin SJ, Eck T, Greshock J, et al: STAC: a method for testing the significance of DNA copy number aberrations across multiple array-CGH experiments. Genome Res 16: 11491158,2006

21. Hauptmann S, Denkert C, Koch I, et al: Genetic alterations in epithelial ovarian tumors analyzed by comparative genomic hybridization. Hum Pathol 33: 632-641, 2002.

22. Mayr D, Hirschmann A, Lohrs U and Diebold J: KRAS and BRAF mutations in ovarian tumors: a comprehensive study of invasive carcinomas, borderline tumors and extraovarian implants. Gynecol Oncol 103: 883-887, 2006.

23. Schraml P, Schwerdtfeger G, Burkhalter F, et al: Combined array comparative genomic hybridization and tissue microarray analysis suggest PAK1 at 11q13.5-q14 as a critical oncogene target in ovarian carcinoma. Am J Pathol 163: 985$992,2003$.

24. Israeli O, Gotlieb WH, Friedman E, et al: Familial vs sporadic ovarian tumors: characteristic genomic alterations analyzed by CGH. Gynecol Oncol 90: 629-636, 2003.

25. Patael-Karasik Y, Daniely M, Gotlieb WH, et al: Comparative genomic hybridization in inherited and sporadic ovarian tumors in Israel. Cancer Genet Cytogenet 121: 26-32, 2000.

26. Van Beers EH, van Welsem T, Wessels LF, et al: Comparative genomic hybridization profiles in human BRCA1 and BRCA2 breast tumors highlight differential sets of genomic aberrations. Cancer Res 65: 822-827, 2005.

27. Wessels LF, van Welsem T, Hart AA, van't Veer LJ, Reinders MJ and Nederlof PM: Molecular classification of breast carcinomas by comparative genomic hybridization: a specific somatic genetic profile for BRCA1 tumors. Cancer Res 62: 7110-7117, 2002

28. Jazaeri AA, Yee CJ, Sotiriou C, Brantley KR, Boyd J and Liu ET: Gene expression profiles of BRCA1-linked, BRCA2linked, and sporadic ovarian cancers. J Natl Cancer Inst 94: 990-1000, 2002.

29. Jonsson G, Naylor TL, Vallon-Christersson J, et al: Distinct genomic profiles in hereditary breast tumors identified by array-based comparative genomic hybridization. Cancer Res 65: 7612-7621, 2005

30. Abdel-Rahman WM, Ollikainen M, Kariola R, et al: Comprehensive characterization of HNPCC-related colorectal cancers reveals striking molecular features in families with no germline mismatch repair gene mutations. Oncogene 24: 1542$1551,2005$.

31. Chan TL, Curtis LC, Leung SY, et al: Early-onset colorectal cancer with stable microsatellite DNA and near-diploid chromosomes. Oncogene 20: 4871-4876, 2001.

32. Schlegel J, Stumm G, Scherthan H, et al: Comparative genomic in situ hybridization of colon carcinomas with replication error. Cancer Res 55: 6002-6005, 1995.

33. Okuda T, Otsuka J, Sekizawa A, et al: P53 mutations and overexpression affect prognosis of ovarian endometrioid cancer but not clear cell cancer. Gynecol Oncol 88: 318-325, 2003.

34. Brown LA, Kalloger SE, Miller MA, et al: Amplification of 11 q13 in ovarian carcinoma. Genes Chromosomes Cancer 47: 481-489, 2008

35. Ratnam K and Low JA: Current development of clinical inhibitors of poly(adp-ribose) polymerase in oncology. Clin Cancer Res 13: 1383-1388, 2007.

36. Serrano-Olvera A, Duenas-Gonzalez A, Gallardo-Rincon D, Candelaria $M$ and De la Garza-Salazar J: Prognostic, predictive and therapeutic implications of HER2 in invasive epithelial ovarian cancer. Cancer Treat Rev 32: 180-190, 2006.

37. Dong Y, Walsh MD, McGuckin MA, et al: Increased expression of cyclin-dependent kinase inhibitor 2 (CDKN2A) gene product p16ink4a in ovarian cancer is associated with progression and unfavourable prognosis. Int J Cancer 74: 57-63, 1997.

38. Niederacher D, Yan HY, An HX, Bender HG and Beckmann MW: CDKN2A gene inactivation in epithelial sporadic ovarian cancer. Br J Cancer 80: 1920-1926, 1999. 
39. Quinn JE, James CR, Stewart GE, et al: BRCA1 mRNA expression levels predict for overall survival in ovarian cancer after chemotherapy. Clin Cancer Res 13: 7413-7420, 2007.

40. Massey A, Offman J, MacPherson P and Karran P: DNA mismatch repair and acquired cisplatin resistance in E. Coli and human ovarian carcinoma cells. DNA Repair (Amst) 2: 73-89, 2003.

41. Samimi G, Fink D, Varki NM, et al: Analysis of MLH1 and MSH2 expression in ovarian cancer before and after platinum drug-based chemotherapy. Clin Cancer Res 6: 1415-1421, 2000 .

42. Lakhani SR, Van De Vijver MJ, Jacquemier J, et al: The pathology of familial breast cancer: predictive value of immunohistochemical markers estrogen receptor, progesterone receptor, HER-2, and p53 in patients with mutations in BRCA1 and BRCA2. J Clin Oncol 20: 2310-2318, 2002.
43. Kurman RJ and Shih Ie M: Pathogenesis of ovarian cancer: lessons from morphology and molecular biology and their clinical implications. Int J Gynecol Pathol 27: 151-160, 2008.

44. Dubeau L: The cell of origin of ovarian epithelial tumours. Lancet Oncol 9: 1191-1197, 2008.

45. Levanon K, Crum C and Drapkin R: New insights into the pathogenesis of serous ovarian cancer and its clinical impact. J Clin Oncol 26: 5284-5293, 2008.

46. Dehari R, Kurman RJ, Logani S and Shih Ie M: The development of high-grade serous carcinoma from atypical proliferative (borderline) serous tumors and low-grade micropapillary serous carcinoma: a morphologic and molecular genetic analysis. Am J Surg Pathol 31: 1007-1012, 2007.

47. Scott M and McCluggage WG: Current concepts in ovarian epithelial tumorigenesis: correlation between morphological and molecular data. Histol Histopathol 21: 81-92, 2006. 